\title{
Louva-a deuses (Dictyoptera, Mantodea) da coleção do Museu da UEFS: o problema da delimitação de espécie no grupo
}

\author{
Vanine Haywanon Santos Maia ${ }^{1}$; Freddy Ruben Bravo Quijano ${ }^{2}$; \\ 1. Bolsista PIBIC/CNPq, Graduando em Nome do Curso, Universidade Estadual de Feira de Santana, e-mail: \\ vaninemaia51@gmail.com \\ 2. Orientador, Departamento de nome, Universidade Estadual de Feira de Santana, e-mail: fbravo@uefs.br
}

PALAVRAS-CHAVE: Insecta, mantódeos, semiárido

\section{INTRODUÇÃO}

Os integrantes da ordem Mantodea Burmeister, 1838 são conhecidos vulgarmente como louva-a-deus (ou louva-deus), põe-mesa (ou ponha mesa), pai-de-cobra, bendito e cavalinho-de-nosso-senhor (Terra \& Agudelo, 2012). São insetos hemimetábolos, terrestres com tamanhos que variam de 8 a $170 \mathrm{~mm}$, são predadores e possuem adaptações morfológicas e anatômicas para tal comportamento. Entre as características mais importantes destacam-se aquelas próprias para a captura das presas: a) presença olhos compostos grandes que oferecem uma visão binocular aperfeiçoada; b) primeiro par de pernas modificado para captura, pernas raptoriais, com fêmures e tíbias prendadas de espinhos o que favorecem a captura da presa; c) pronoto alongado, lugar este onde as pernas raptoriais se posicionam no momento da inércia; d) coloração que favorece a camuflagem contra predadores e aparelho de armadilha das presas (Figura 1) (Grimaldi \& Engel 2005).

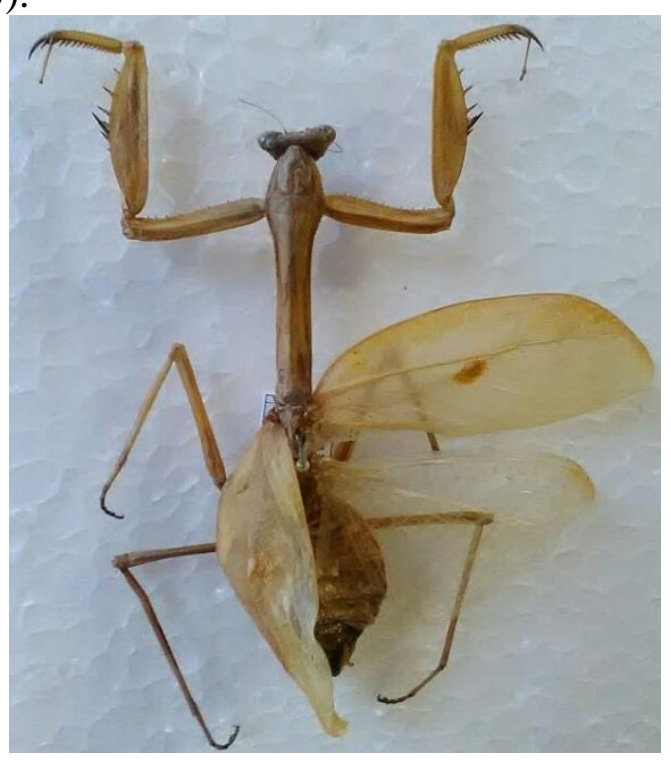

Figura1 Stagmatoptera binotata, habitus dorsal

A ordem Mantodea é cosmopolita em sua distribuição e é classificada em 17 famílias, sete das quais são encontradas na região Neotropical e seis são registradas no Brasil: Acanthopidae, Chaeteessidae, Liturgusidae, Mantidae, Mantoididae e Thespidae (Terra \& Agudelo, 2012; Menezes \& Bravo, 2014); Hymenopodidade, a outra família Neotropical é encontrada somente no Caribe. São conhecidas no mundo aproximadamente 2.400 espécies/subespécies de mantódeos (Ehrmann, 2002), e desse total apenas $20 \%$ são encontrados na região Neotropical, que correspondem a 498 espécies (Rivera 2010). No Brasil são conhecidas 244 espécies e 68 gêneros (Agudelo, 2016), ou seja, quase a metade das conhecidas para a região Neotropical. A riqueza 
estimada para o Brasil de mantódeos é de 700 espécies (Terra \& Aguedelo, 2012), ou seja, atualmente seriam conhecidas menos da metade de espécies dessa ordem para o país. No Semiárido brasileiro foram registradas todas as famílias brasileiras de Mantódea, 36 espécies e 26 gêneros (Menezes \& Bravo, 2013).

Na coleção Prof. Johann Becker do Museu de Zoologia da Universidade Estadual de Feira de Santana (MZFS) estão depositados mais de 500 exemplares, a maioria deles montados em alfinete entomológico e ao menos 250 em álcool $70 \%$; estes últimos exemplares devem ser preparados para montagem para sua posterior identificação. Cerca de $40 \%$ dos exemplares estão identificados até espécie.

A quase totalidade desses exemplares foram coletados em diversas localidades do semiárido brasileiro do projeto PPBio (Programa de Pesquisa em Biodiversidade). A taxonomia de Mantodea tem se caracterizado pela aderência a um definição ampla de espécie (visão lumper em taxonomia) o que leva a incluir grande variação morfológica na delimitação de uma espécie. A outra variante em taxonomia, splitter, considera uma definição mais exata e restrita das categorias taxonômicas, e no caso específico de espécie.

Desta maneira, este plano de trabalho visa trabalhar com um grupo de insetos com grande representatividade no Museu de Zoologia da UEFS que precisam ser identificados e, ainda, discutir se uma delimitação de espécie mais ampla (lumper) ou mais estrita (splitter) pode ter sustento com análises de análises de séries grandes de uma espécie.

\section{MATERIAL E MÉTODOS}

Foram estudados os espécimes alfinetados a seco e armazenados em álcool $70 \%$ procedentes de várias localidades do Semiárido brasileiro, depositados na Coleção Entomológica Professor Johann Becker do Museu de Zoologia da Universidade Estadual de Feira de Santana (MZFS). Os exemplares em álcool 70\% foram preparados e montados em alfinete entomológico, seguindo a recomendação de Agudelo \& Chica (2002). Uma vez secos na estufa a $42^{\circ} \mathrm{C}$ por sete dias, os espécimes serão etiquetados e serão tombados no Museu de Zoologia da UEFS (MZFS).

Para a identificação dos espécimes foram usadas as chaves de identificação para gênero de Agudelo \& Chica (2002).

\section{RESULTADOS E DISCUSSÃO}

Foram estudados 500 exemplares depositados na Coleção Entomológica Prof. Johann Becker do Museu de Zoologia da Universidade Estadual de Feira de Santana (MZFS). Grande parte desse material foi coletado em várias localidades do Semiárido brasileiro, pelo projeto PPBio (Programa de Pesquisa em Biodiversidade). Uma parte dos exemplares está montado em alfinete entomológico e $40 \%$ deles estão identificados até espécie.

Uma quantidade menor desses 500 exemplares, 250 em total, estavam armazenados em álcool, 101 dos quais foram montados e identificados até gênero, ou seja, aproximadamente $70 \%$ usando a chave de identificação de Agudelo \& Chica (2002).

Foram identificados exemplares de cinco famílias no Semiárido: Chaeteessidae, Mantoididae, Acanthopidae, Thespidae e Mantidae. De 250 espécimes em álcool, foram montados 85 espécimes de Mantidae, 12 de Thespidae, quatro de Acanthopidae. Além disso, foram identificados até gênero 92 espécimes da família Mantoididae, e 16 exemplares da família Thespidae. 
Os exemplares eram de localidades como, Rio Grande do Norte, Paraíba, Bahia, Pernambuco, Ceará, sendo que a maioria deles são provenientes da Bahia, de regiões do Semiárido.

Antes desse projeto de pesquisa houve um registro na literatura de 26 gêneros e 36 espécies de Mantodea para o Semiárido (Menezes \& Bravo 2013), comparando esses dados com os resultados obtidos no projeto pode se observar que não houve um aumento de espécies ou gêneros novos conhecidos para o Semiárido.

\section{REFERÊNCIAS}

AGUDELO, A. \& CHICA, L. 2002. Mantidos: Introducción al Conocimento del Orden Mantodea. Universidad Distrital Francisco José de Caldas, Centro de Investigación y Desarrollo Científico, Bogotá, 90 p.

EHRMANN, R. 2002. Gottesanbeterinnen der Welt. Munster: Natur und Tier Verlag, 519 pp.

MENEZES, E. C. \& BRAVO, F, 2014. Capítulo 9. Mantodea (Insecta) do Semiárido. In Bravo. F \& Calor (orgs). Artrópodes do Semiárido: Biodiversidade e Conservação, Print Mídia. Feira de Santana. pp. 111-116.

GIGLIO-TOS, E. 1927. Orthoptera-Mantidae. Das tierreich. Walter de Gruyter \& Co., Berlin, 50, 707p.

GRIMALDI, D.A. \& ENGEL, M.S. 2005. Evolution of the Insects. Cambridge University Press, New York, 784 pp.

MENEZES, E.C. \& F. BRAVO. 2013. Uma nova espécie de Orthoderella Giglio-Tos (Mantodea, Mantidae, Photinainae) do Brasil. Revista Brasileira de Entomologia 57: 12-18.

RIVERA, J. 2010. A historical review of praying mantid taxonomy and systematics in the Neotropical Region: State of Knowledge and recente advances (Insecta: Mantodea). Zootaxa, 2638:44-64

TERRA, P. S. \& AGUDELO, A. A. 2012. Mantodea, p.323-331. In: J.A. Rafael, G.A. R. MELO, C.J. B. de CARVALHO, S.A. CASARI \& R.CONSTANTINO (eds.). Insetos do Brasil. Diversidade e Taxonomia. Manaus, Holos Editora. 810p. TERRA, P.S. 1995. Revisão Sistemática dos Gêneros de Louva-a-Deus da região Neotropical. (Mantodea). Revista Brasileira de Entomologia, 39(1), 13-94. 